\title{
MACC1: A potential molecule associated with pancreatic cancer metastasis and chemoresistance
}

\author{
GANG WANG* ${ }^{*}$ MU-XING KANG* ${ }^{*}$ WEN-JIE LU, YING CHEN, BO ZHANG and YU-LIAN WU \\ Department of Surgery, The Second Affiliated Hospital, College of Medicine, \\ Zhejiang University, Cancer Institute of Zhejiang University, \\ Hangzhou, Zhejiang 310009, P.R. China
}

Received February 14, 2012; Accepted May 11, 2012

DOI: $10.3892 / \mathrm{ol} .2012 .784$

\begin{abstract}
It has been suggested that the newly identified metastasis-associated in colon cancer-1 (MACC1) oncogene is involved in the progression and metastasis of cancer. Several studies have indicated that MACC1 has potential as a novel biomarker. In this study, we aimed to investigate the functions and serum expression levels of MACC1 in pancreatic cancer patients. Blood serum samples from 60 cancer patients and 49 controls were analyzed for serum MACC1 by ELISA. The results revealed that high expression levels of MACC1 were correlated with lymph node metastasis, distant metastasis and a later TNM stage. Inhibition of MACC1 by siRNAs significantly suppressed pancreatic cancer cell proliferation and migration. Furthermore, it was found that the downregulation of MACC1 sensitized pancreatic cancer cells to gemcitabine treatment through the inhibition of the Ras/ERK signaling pathway. Our findings suggest that MACC1 may aid in the diagnosis of pancreatic cancer and serve as a potential therapeutic target.
\end{abstract}

\section{Introduction}

Pancreatic cancer is one of the most aggressive malignancies worldwide. It is ranked as the fourth leading cause of cancer mortality in the United States and the sixth leading cause of cancer mortality in China (1). A 2011 analysis of the global incidence of cancer mortality revealed that there are approximately 138,100 and 127,900 annual male and female mortalities worldwide, respectively (2). Due to its high propensity for local invasion, distant metastases and a lack

Correspondence to: Dr Yu-Lian Wu, Department of Surgery, The Second Affiliated Hospital, College of Medicine, Zhejiang University, Cancer Institute of Zhejiang University, 88 Jiefang Road, Hangzhou, Zhejiang 310009, P.R. China

E-mail:wuyulian@medmail.com.cn

${ }^{*}$ Contributed equally

Key words: metastasis-associated in colon cancer-1, metastasis, epithelial-mesenchymal transition, chemoresistance of early detection methods when diagnosed, more than twothirds of cases demonstrate advanced or unresectable forms of the disease. Thus, the five-year survival rate for pancreatic cancer patients remains less than 5\% (3). The pathogenesis of pancreatic cancer is a complex, multistage and multigenetic process, of which the precise mechanisms are not yet fully understood. Gemcitabine, a cytotoxic nucleoside analogue, is the current therapeutic strategy for advanced pancreatic cancer, but it provides no significant survival advantage to patients (4). Accordingly, there is great demand to identify novel oncogenes and clinically applicable molecular targets for the diagnosis and treatment of this disease (5).

The metastasis-associated in colon cancer-1 (MACC1) gene was identified by a genome-wide search for genes differentially expressed by analyzing normal tissues, primary tumors and metastastic lesions in colon cancer (6). MACC1 has been found to induce tumor proliferation, invasion and metastasis in cell culture, as well as in patients with colon cancer. Moreover, MACC1 expression is upregulated at the crucial step of transition from adenoma to carcinoma. Furthermore, in distant metastases, MACC1 mainly translocates into the nucleus from the cytoplasm. Notably, hepatocyte growth factor (HGF) treatment may also cause MACC1 nuclear translocation and following this, MACC1 binds to the promoter of the receptor tyrosine kinase Met and activates its transcription. The resulting upregulation of Met enhances the HGF/ Met pathway and MACC1 acts as a key regulator (7). MACC1 may be a target gene of the MAPK signaling pathway (6). MACC1 was reported to be elevated in various cancer tissues, including colon cancer $(6,8)$, hepatocarcinoma (9) and lung adenocarcinoma $(10,11)$, and upregulation of its gene expression was also observed in gastric cancer (12). However, until recently, there has been no study demonstrating that serum MACC1 may be used as a biomarker in cancer, and little is known regarding its role in pancreatic cancer development and drug resistance.

In the present study, we have focused on evaluating the clinical significance of serum MACC1 for the diagnosis of pancreatic cancer. In addition to this, we investigated the effect of MACC1 inhibition on pancreatic cancer proliferation, metastasis and sensitivity to gemcitabine. We also explored the possible underlying mechanisms that may be involved in gemcitabine resistance induced by MACC1. 


\section{Patients and methods}

Patients and serum. A total of 109 serum samples were analyzed. The study included 60 patients with pancreatic cancer and 49 healthy controls who were hospitalized in the Department of Surgery at The Second Affiliated Hospital of Zhejiang University, Zhejiang, China, between January 2010 and December 2011. The serum samples had been obtained the day before surgical procedures or before the initiation of neoadjuvant chemotherapy, and stored at $-80^{\circ} \mathrm{C}$ until analysis. The clinical characteristics of the patients, including gender, age and TNM stage are summarized in Table I. The clinical stage of the tumors was determined according to the Union for International Cancer Control (UICC) 2002 TNM staging of pancreatic cancer (13). The present study was approved by the Ethical Committee within our hospital and informed consent was obtained from each patient prior to surgery or collection of blood samples.

Measurement of MACC1 in serum samples. The serum levels of MACC1 were measured by ELISA (Uscn Life Science Inc., Wuhan, China) according to the manufacturer's instructions, and all measurements were conducted in duplicate. A $100 \mu 1$ measurement of each dilution of standard (provided by the manufacturer), sample and blank was added to a 96-well plate that had been coated with MACC1 antibody and incubated for $2 \mathrm{~h}$ at $37^{\circ} \mathrm{C}$. Each well was aspirated and subsequently washed with washing buffer (provided by the manufacturer). Following this, $100 \mu 1$ of detection reagent $\mathrm{A}$ was added to each well and the plate was incubated for 1 $\mathrm{h}$ at $37^{\circ} \mathrm{C}$. Once any unbound antibody had been removed, $100 \mu 1$ of detection reagent $\mathrm{B}$ was added to each well and the plate was incubated at $37^{\circ} \mathrm{C}$ for $30 \mathrm{~min}$. Following the washing process, $90 \mu 1$ of substrate solution was added to the wells and the plate was incubated for $20 \mathrm{~min}$ at $37^{\circ} \mathrm{C}$ in a dark environment. The reaction was then terminated with $50 \mu 1$ stop solution. The optical density of each well was determined immediately using a microplate reader at $450 \mathrm{~nm}$. Standard curves generated by a two-parameter logistic curve fit analysis were used to determine the MACC1 concentrations of the samples.

Cell culture and reagents. The human pancreatic cancer cell lines (CFPAC-1, AsPC-1, PANC-1, MIA PaCa-2 and BxPC-3) and the human colon cancer cell lines (SW480 and SW620) were purchased from the American Type Culture Collection (Manassas, VA, USA). CFPAC-1 cells were maintained in Iscove's Modified Dulbecco's Medium (IMDM), PANC-1 and MIA PaCa-2 cells were maintained in Dulbecco's modified Eagle's medium and AsPC-1, BxPC-3, SW480 and SW620 cells were maintained in RPMI-1640 medium. All media were supplemented with $10 \%$ fetal bovine serum (FBS), $100 \mathrm{U} / \mathrm{ml}$ penicillin and $100 \mathrm{mg} / \mathrm{ml}$ streptomycin. The cells were grown in a monolayer culture in a humidified incubator containing $5 \% \mathrm{CO}_{2}$ in air at $37^{\circ} \mathrm{C}$. Gemcitabine was purchased from Eli Lilly (Bad Homburg, Germany) and dissolved in sterile $0.9 \%$ sodium chloride to obtain a stock solution.

siRNA transfection. Three siRNA sequences targeting human MACC1 and a negative control siRNA were obtained from
Shanghai Genepharma Co., Ltd. (Shanghai, China). The sequences of MACC1 and control siRNA were as follows: MACC1 siRNA1 sense, 5'-CAGGCUAUUUGCAGAA GAATT-3' and antisense, 5'-UUCUUCUGCAAAUAGCCU GTT-3'; MACC1 siRNA2 sense, 5'-GAGCAAGUAAU GUUUAUGUTT-3 and antisense, 5'-ACAUAAACAUU ACUUGCUCTT-3'; MACC1 siRNA3 sense, 5'-GCAGUGC UAAGACAAAGCATT-3' and antisense, 5'-UGCUUUGUC UUAGCACUGCTT-3'; negative control siRNA sense, 5'-UUC UCCGAACGUGUCACGUTT-3' and antisense, 5'-ACGUGA CACGUUCGGAGAATT-3'. The cells were seeded in 6-well cell culture plates at a density of $2 \times 10^{5}$ cells/well and cultured to $40 \%$ confluence on the day before transfection. siRNA transfection was performed using Lipofectamine 2000 (Invitrogen, Carlsbad, CA, USA) according to the manufacturer's instructions. The effects of the transfection on MACC1 reduction were assayed by western blot analysis and flow cytometry analysis.

RNA preparation and quantitative real-time PCR ( $q R T-P C R)$. Total RNA was isolated from cell cultures with TRIzol reagent (Invitrogen) according to the manufacturer's instructions. RNA concentrations were determined by spectrophotometry. Complementary DNA was synthesized from $5 \mu \mathrm{g}$ of total RNA using M-MLV Reverse Transcriptase (Promega, Madison, WI, USA). The expression of MACC1 mRNA relative to glyceraldehyde-3-phosphate dehydrogenase (GAPDH) was measured 3 times by qRT-PCR using the Light Cycler real-time PCR system (Roche, Mannheim, Germany). The primers for MACC1 and GAPDH were as follows: MACC1 forward, 5'-TCTGTATGAACTTATTGTGGCTC-3' and reverse, 5'-CATAGGCAGGTTTCCACATC-3'; GAPDH forward, 5'-TGCACCACCAACTGCTTAG-3' and reverse, 5'-GAGGCAGGGATGATGTTC-3'.

Cell proliferation assay. Cell viability was determined using the 3-(4,5-dimethyl-thiazol-2-yl)-2,5-diphenyltetrazolium bromide (MTT) assay (Sigma, St. Louis, MO, USA). Cells were plated in a 96-well plate at 3000 cells/well. Following this, $20 \mu 1$ of MTT solution $(5 \mathrm{mg} / \mathrm{ml})$ was added to each well and the plate was incubated at $37^{\circ} \mathrm{C}$ for $4 \mathrm{~h}$. The medium was then discarded, and the purple-blue MTT formazan precipitate was dissolved in $150 \mu 1$ dimethyl sulfoxide. The plates were mixed for $10 \mathrm{~min}$ on a gyratory shaker and absorbance at $570 \mathrm{~nm}$ was measured by an ELISA reader (ELx800; Bio Tek Instruments, Inc., Winooski, VT, USA).

Migration assay. The migration assays were performed in 24-well plates using transwell filters with $8.0 \mu \mathrm{m}$ pore size and $6.5 \mathrm{~mm}$ diameter (Corning Inc., Corning, NY, USA). On the day following transfection, CFPAC- 1 cells were trypsinized and seeded at a density of $1 \times 10^{5}$ cells/well. The upper chamber contained $300 \mu 1$ of IMDM $(0.5 \%$ FBS $)$ and the lower chamber contained $600 \mu \mathrm{l}$ of IMDM (10\% FBS). Following 48-h incubation, the cells were fixed with $4 \%$ paraformaldehyde for $10 \mathrm{~min}$ and stained with eosin for $10 \mathrm{~min}$. The non-migrated cells on the upper side of the membranes were removed using wet cotton swabs. The stained cells on the lower side of the membranes were counted in at least 5 fields under the microscope at a magnification of $\mathrm{x} 200$. 
Table I. Correlation between serum MACC1 expression levels and clinicopathological parameters of pancreatic cancer.

\begin{tabular}{|c|c|c|c|c|c|c|c|}
\hline \multirow[b]{2}{*}{$\begin{array}{l}\text { Clinicopathological } \\
\text { parameters }\end{array}$} & \multirow[b]{2}{*}{ No. of cases } & \multicolumn{4}{|c|}{ MACC1 expression } & \multirow[b]{2}{*}{$\chi^{2}$} & \multirow[b]{2}{*}{ P-value } \\
\hline & & Low & $\%$ & High & $\%$ & & \\
\hline Gender & & & & & & 0.141 & 0.707 \\
\hline Female & 20 & 13 & 65.0 & 7 & 35.0 & & \\
\hline Male & 40 & 24 & 60.0 & 16 & 40.0 & & \\
\hline Age (years) & & & & & & 0.984 & 0.321 \\
\hline$\leq 60$ & 23 & 16 & 69.6 & 7 & 30.4 & & \\
\hline$>60$ & 37 & 21 & 56.8 & 16 & 43.2 & & \\
\hline Invasion depth & & & & & & 0.242 & 0.623 \\
\hline $\mathrm{T} 1+\mathrm{T} 2$ & 11 & 8 & 72.7 & 3 & 27.3 & & \\
\hline $\mathrm{T} 3+\mathrm{T} 4$ & 49 & 29 & 59.2 & 20 & 40.8 & & \\
\hline Lymph node metastasis & & & & & & 4.467 & 0.035 \\
\hline No & 14 & 12 & 85.7 & 2 & 14.3 & & \\
\hline Yes & 46 & 25 & 54.3 & 21 & 45.7 & & \\
\hline Distant metastasis & & & & & & 7.944 & 0.005 \\
\hline No & 24 & 20 & 83.3 & 4 & 16.7 & & \\
\hline Yes & 36 & 17 & 47.2 & 19 & 52.8 & & \\
\hline TNM stages & & & & & & 5.107 & 0.024 \\
\hline $\mathrm{I}+\mathrm{II}$ & 18 & 15 & 83.3 & 3 & 16.7 & & \\
\hline III+IV & 42 & 22 & 52.4 & 20 & 47.6 & & \\
\hline
\end{tabular}

MACC1, metastasis-associated in colon cancer-1.

Western blot analysis. The cultured cells were harvested and lysed in cold RIPA lysis buffer (Beyotime Institute of Biotechnology, Jiangsu, China) supplemented with a complete protease inhibitor cocktail (Roche) composed of $50 \mathrm{mmol} / 1 \mathrm{NaF}$ and $1 \mathrm{mmol} / 1 \mathrm{Na}_{3} \mathrm{VO}_{4}$. The supernatants were separated by centrifugation at $12,000 \mathrm{x} \mathrm{g}$ for $20 \mathrm{~min}$ at $4^{\circ} \mathrm{C}$, and the protein concentrations were determined using a BCA Protein Assay kit (Thermo, USA). Following this, $40 \mathrm{mg}$ of protein was resolved by electrophoresis on 8-12\% SDS-PAGE and transferred onto polyvinylidene difluoride (PVDF) membranes (Bio-Rad, Hercules, CA, USA). The transblotted membranes were blocked with TBST containing 5\% skimmed milk for $2 \mathrm{~h}$ at room temperature, and then incubated with the appropriate antibodies. Antibodies used in this study were as follows: MACC1 (Abcam, Cambridge, MA, USA), Bcl-2, caspase-3, caspase-9, E-cadherin, vimentin, $\beta$-actin (Cell Signaling Technology, Inc., Danvers, MA, USA), PARP-1, Gab2, p-ERK1/2, ERK1/2 (Santa Cruz Biotechnology, Inc., Santa Cruz, CA, USA) and Ras (Epitomics, Inc., Burlingame, CA, USA). The secondary antibodies were purchased from Beijing Zhongshan Golden Bridge Biotechnology Co., Ltd., Beijing, China. Bands were visualized using an enhanced chemiluminescence (ECL) reagent kit (Beyotime) and exposed to X-ray film.

Flow cytometric analysis of apoptosis. CFPAC-1 cells $\left(2 \times 10^{5}\right)$ were seeded in 6-well plates for $24 \mathrm{~h}$ and transfected with siRNA. Following transfection, CFPAC-1 cells were treated with or without $0.5 \mu \mathrm{g} / \mathrm{ml}$ gemcitabine for $48 \mathrm{~h}$. The CFPAC-1 cells were collected, washed with phosphate-buffered saline (PBS) containing $0.1 \%$ bovine serum albumin and re-suspended in $500 \mu \mathrm{l}$ binding buffer. Following this, the cells were incubated with $5 \mu \mathrm{l}$ annexin V-FITC and $10 \mu \mathrm{l}$ PI solution for $15 \mathrm{~min}$ at room temperature in a dark environment. Subsequently, the samples were evaluated for apoptosis using flow cytometry (FACSCalibur; BD Biosciences, Franklin Lakes, NJ, USA).

Statistical analysis. Each experiment was performed in triplicate. The data were shown as the mean \pm standard deviation (SD). All statistical tests were performed using Statistical Program for Social Sciences (SPSS) software 18.0 (SPSS Inc., Chicago, IL, USA). Correlations between MACC1 expression and clinicopathological features were analyzed using the $\chi^{2}$ test. $\mathrm{P}<0.05$ was considered to indicate a statistically significant difference.

\section{Results}

Correlations between serum MACC1 levels and clinicopathological features of pancreatic cancer patients. The serum samples from 60 patients with pathologically confirmed pancreatic cancer and 49 healthy controls were analyzed. The demographic characteristics of the patients are summarized in Table I. We measured serum MACC1 levels using a commercial ELISA kit. To ensure that the immunoassay was suitable 
for detecting clinical serum samples, the linearity was examined to demonstrate excellent linearity with serial dilutions. The minimum detectable dose of human MACC1 measured by this kit was typically $<0.312 \mathrm{ng} / \mathrm{ml}$. The MACC1 levels in the healthy subjects were too low to be measured precisely using this ELISA kit. In our study, when the MACC1 level was $<0.312 \mathrm{ng} / \mathrm{ml}$ it was considered negative and when $>0.312 \mathrm{ng} /$ $\mathrm{ml}$ it was considered positive. To determine the association between the serum MACC1 levels and clinical characteristics, patients were divided into low level $(<4 \mathrm{ng} / \mathrm{ml})$ and high level ( $\geq 4 \mathrm{ng} / \mathrm{ml}$ ) group. We determined the cut-off level according to the 'minimum P-value approach' (14). The rate of positive MACC1 expression was $63.3 \%$ (38/60) in the pancreatic cancer patients and $4.1 \%(2 / 49)$ in the healthy subjects (Fig. 1). A significant difference in the level of MACC1 expression was demonstrated between the pancreatic cancer patients and the healthy subjects $\left(\chi^{2}=40.763 ; \mathrm{P}<0.001\right)$. Table I shows the correlations between the serum MACC1 level and the clinicopathological characteristics of individuals with pancreatic cancer. In these patients, no significant difference was found in serum MACC1 levels according to gender $(\mathrm{P}=0.707)$, age $(\mathrm{P}=0.321)$ and invasion depth $(\mathrm{P}=0.623)$. However, a high level of serum MACC1 expression was positively correlated with lymph node metastasis $(\mathrm{P}=0.035)$, distant metastasis $(\mathrm{P}=0.005)$ and later TNM stages $(\mathrm{P}=0.024)$. These data indicated that serum MACC1 may be a novel diagnostic marker for pancreatic cancer.

Expression of MACC1 in pancreatic cancer cell lines. Previous studies have indicated that MACC1 demonstrates overexpression in SW620 cells and almost no expression in SW480 cells, despite SW60 cells being derived from a metastasis of the same tumor from which the SW480 cell line is derived (6). Consequently, we examined the expression of MACC1 in 5 human pancreatic cancer cell lines. The MACC1 protein expression was high in CFPAC-1 cells, which are derived from a metastatic lesion of ductal adenocarcinoma, and low in BxPC-3 cells. The remaining 3 pancreatic cancer cell lines demonstrated almost no MACC1 expression (Fig. 2A). The human colon cancer cell line, SW480, was used as the negative control and the SW620 cell line as the positive control. We also examined the expression at the mRNA level using qRT-PCR. The qRT-PCR amplification results demonstrate that MACC1 mRNA was not detected in the AsPC-1, PANC-1 and MIA PaCa- 2 cell lines. The mRNA expression of MACC1 in BxPC-3 cells was low, whereas a high expression was noted in the highly metastatic pancreatic cancer cell line CFPAC-1, second to that in the SW620 cell line (Fig. 2B). In comparison with non-metastatic cell lines, the metastatic cell lines presented high levels of MACC1, indicating that MACC1 had a high correlation with metastasis. Therefore, a CFPAC-1 cell line was selected for the subsequent experiments.

MACC1 inhibition by siRNA in CFPAC-1 cells. The present study focused on investigating the potential functions of MACC1 in pancreatic cancer cells. The MACC1 silencing experiment was conducted using 400 pmol of MACC1-siRNA, and the effects of transfection were confirmed $72 \mathrm{~h}$ later by qRT-PCR and western blot analysis. Our data indicated that in

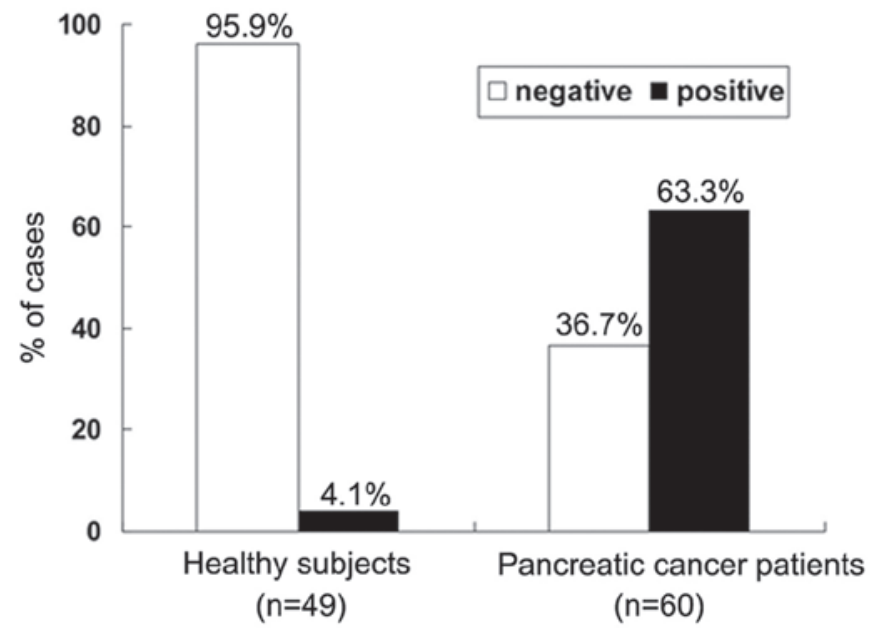

Figure 1. Frequency of serum MACC1 in pancreatic cancer patients and healthy subjects as evaluated using ELISA methods. An optimal cut-off value was identified, MACC1 levels $<0.312 \mathrm{ng} / \mathrm{ml}$ were defined as negative and $>0.312 \mathrm{ng} / \mathrm{ml}$ as positive. MACC1, metastasis-associated in colon cancer-1 .

A
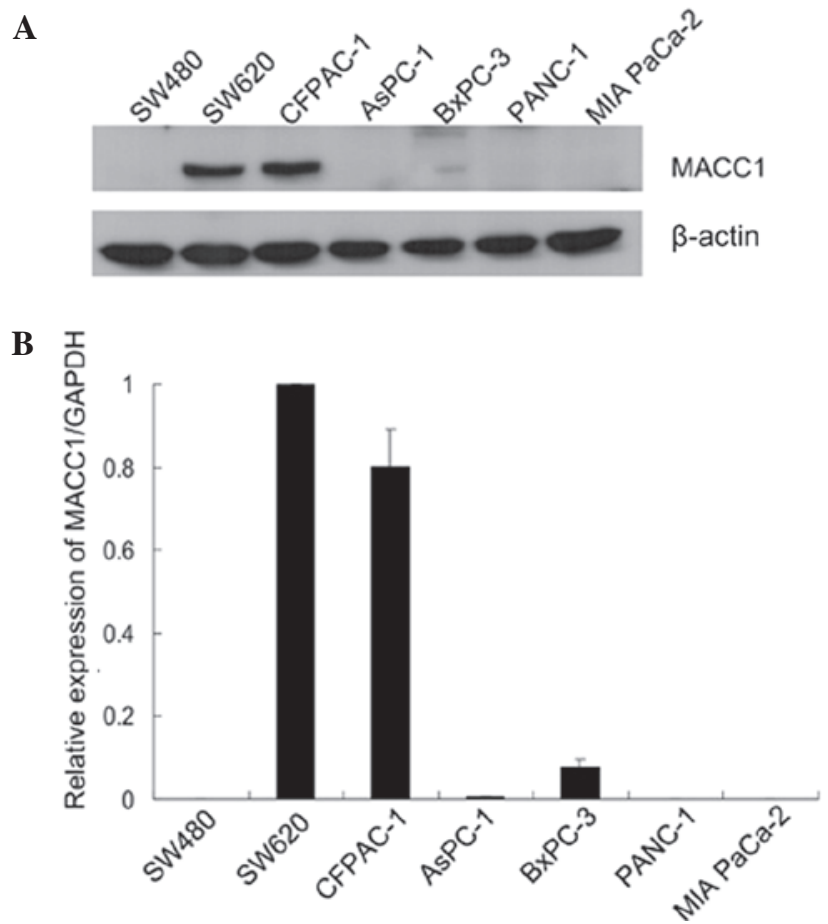

Figure 2. Expression of MACC1 protein in colon cancer and pancreatic cancer cell lines. (A) Western blot analysis of MACC1 expression in colon cancer and pancreatic cancer cell lines. $\beta$-actin served as a loading control. (B) Quantitative real-time PCR analysis of expression of MACC1 in cancer cell lines. The expression of MACC1 in CFPAC-1 cells was similar to that in SW620 cells, derived from a metastatic colon cancer. MACC1, metastasis-associated in colon cancer-1; GAPDH, glyceraldehyde-3-phosphate dehydrogenase.

comparison to the normal control and mock-transfected cells, the expression of MACC1 was reduced in the 3 siRNA-transfected cells, particularly in the MACC1-siRNA2-transfected cells (Fig. 3A). The results were consistent with the experimental data of qRT-PCR (Fig. 3B) where MACC1-siRNA2 
A

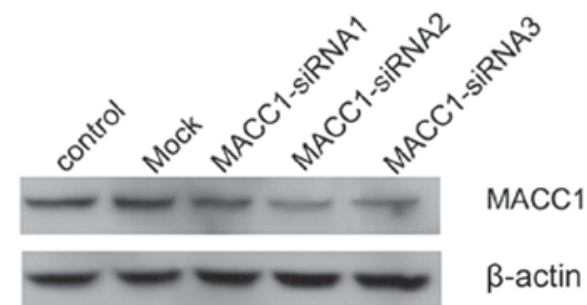

B

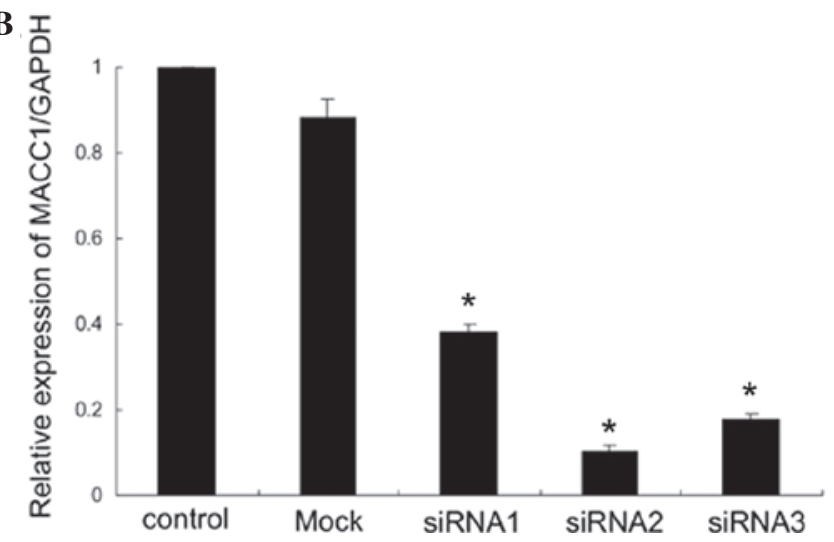

Figure 3. RNA interference suppressed MACC1 expression in CFPAC-1 cells. (A) CFPAC-1 cells were transfected with indicated siRNAs for $72 \mathrm{~h}$ and then collected for western blot analysis. $\beta$-actin served as a loading control. (B) Changes in the mRNA expression of MACC1 followed by siRNA treatment were determined using quantitative RT-PCR. MACC1 siRNA2 was the most effective and was selected for the subsequent experiments Data were shown as the mean \pm SD of three independent experiments. ${ }^{*} \mathrm{P}<0.05$, versus control and mock group. MACC1, metastasis-associated in colon cancer-1; GAPDH, glyceraldehyde-3-phosphate dehydrogenase; $\mathrm{SD}$, standard deviation.

provided the highest inhibitory rate. Downregulation of MACC1 was also visible 4 days following the initial siRNA transfection. In the following experiments, MACC1-siRNA2 was used to downregulate the expression of MACC1 in CFPAC-1 cells.

Downregulation of MACC1 suppressed pancreatic cancer cell proliferation, migration and inhibited epithelial-mesenchymal transition (EMT). To evaluate the effects of MACC1 downregulation on CFPAC-1 cell growth, normal, mock-transfected and siRNA2-transfected cells were seeded in 96-well plates at a density of 3000 cells/well and their growth was monitored using an MTT assay for the following 4 days. In comparison with the control cells, the mock-transfected cells produced a similar growth rate, but the MACC1-siRNA2-transfected cells exhibited a significantly lower growth rate (Fig. 4), indicating that the inhibition of MACC1 may suppress the proliferation of CFPAC-1 cells. To examine the effects of MACC1-siRNA on cell migration, a transwell chamber assay was performed. Following a $48 \mathrm{~h}$ incubation period, the number of migrated cells in the control group was $374 \pm 18$, the mock-transfected group was $331 \pm 17$ and the MACC1-siRNA2 transfected group was $20 \pm 8$. Therefore, there was a reduction in the migration rate of the MACC1-siRNA2 group in comparison with the control and mock-transfected groups following MACC1 inhibition by $95 \%$ and $94 \%$, respectively (Fig. 5A and B). Western blot analyses were performed to confirm that the EMT phenotype was suppressed by MACC 1 inhibition in CFPAC-1 cells

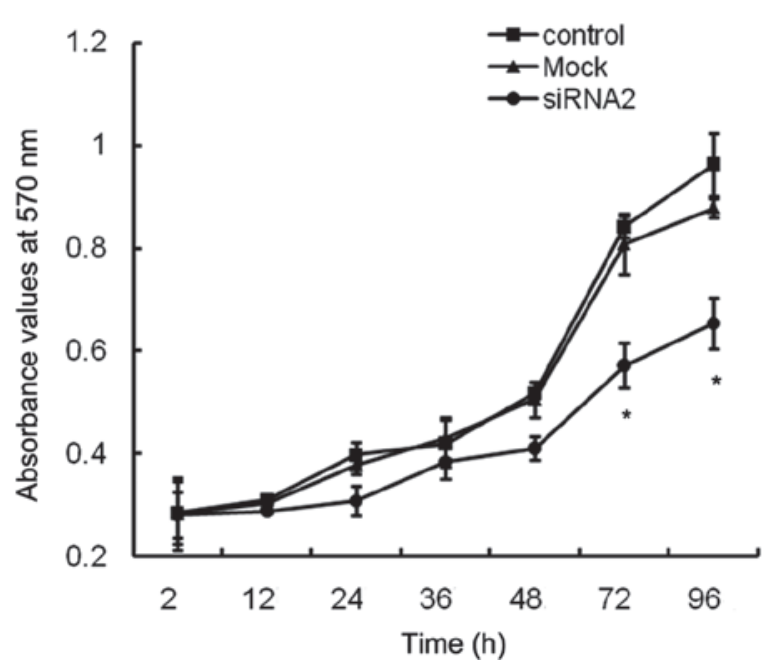

Figure 4. Effect of MACC1 siRNA on CFPAC-1 proliferation. Cell growth curves of CFPAC-1 cells transfected with or without siRNA were determined at various times using an MTT assay. Cell proliferation was significantly inhibited by MACC1 siRNA. Results are shown as the mean \pm SD from three independent experiments. ${ }^{*} \mathrm{P}<0.05$. MACC 1 , metastasis-associated in colon cancer-1; SD, standard deviation.

Following $48 \mathrm{~h}$ of transfection, the level of E-cadherin protein was significantly upregulated in MACC1-siRNA2-transfected cells relative to the control and mock-transfected cells, while the vimentin level did not appear to change (Fig. 5C). Collectively, these results indicated that MACC1 was involved in the EMT of pancreatic cancer cells and its downregulation may inhibit EMT and suppress cancer cell migration.

Downregulation of MACC1 sensitized CFPAC-1 cells to gemcitabine. At present, the best chemotherapeutic agent available for the treatment of advanced pancreatic cancer is gemcitabine (15). To verify that MACC1 was directly involved in the mediation of chemoresistance, CFPAC- 1 cells were transfected with siRNA against MACC1 to downregulate its expression prior to gemcitabine treatment. MACC1-siRNA2 was used in the subsequent experiments and cell viability was evaluated using the MTT assay. Following $24 \mathrm{~h}$ of gemcitabine incubation, no significant difference in the inhibitory rate was observed among the 3 groups. However, following $48 \mathrm{~h}$ of gemcitabine incubation, there was a significant growth inhibition in MACC1-siRNA2-transfected cells in comparison with the control and mock-transfected cells at the concentrations of $0.5 \mu \mathrm{g} / \mathrm{ml}$ and $5 \mu \mathrm{g} / \mathrm{ml}$, respectively (Fig. 6A). Following $72 \mathrm{~h}$ of gemcibatine incubation at these concentrations, MACC1 downregulation resulted in an increased inhibitory effect on the proliferation of CFPAC-1 cells. Furthermore, we investigated the degree of cell death in response to gemcitabine treatment in the 3 cell groups using flow cytometry. In absence of gemcitabine, suppression of MACC1 caused a slight reduction in surviving cells and an increased percentage of apoptotic and dead cells. Following incubation with gemcitabine $(0.5 \mu \mathrm{g} / \mathrm{ml})$ for $48 \mathrm{~h}$, MACC1 downregulation resulted in a strong apoptotic effect. The percentage of live cells in the MACC1-siRNA-transfected, control and mock-transfected groups was 29.7, 47.3 and $49.5 \%$, 
A

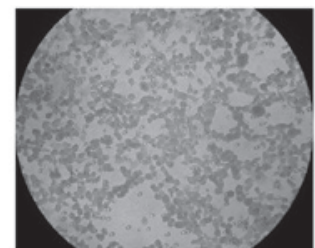

control

B

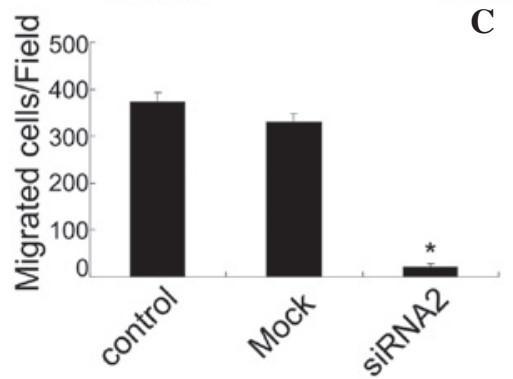

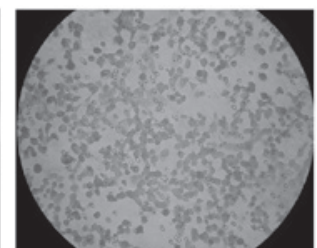

Mock

C

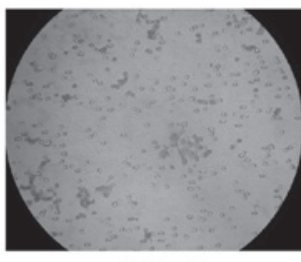

siRNA2

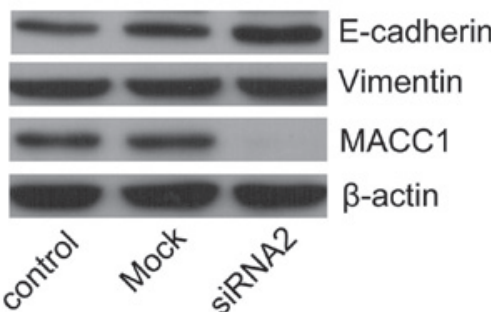

Figure 5. Downregulation of MACC1 suppressed CFPAC-1 cell migration and inhibited EMT. (A) CFPAC-1 cells transfected with or without siRNA were reseeded in migration transwell chambers as indicated in Patients and methods. The migrated cells were stained $48 \mathrm{~h}$ later, counted and visualized on an optical microscope (magnification, x200). (B) Numbers of migrated cells in each group. Data were shown as the mean \pm SD of 5 fields. Compared to the control and mock groups, the migrated cells in the siRNA2 group were significantly decreased. ${ }^{*} \mathrm{P}<0.05$. (C) Western blot analysis of MACC1, E-cadherin and vimentin in CFPAC-1 cells transfected with or without siRNA. $\beta$-actin served as a loading control. MACC1, metastasis-associated in colon cancer-1; EMT, epithelial-mesenchymal transition; SD, standard deviation.

A
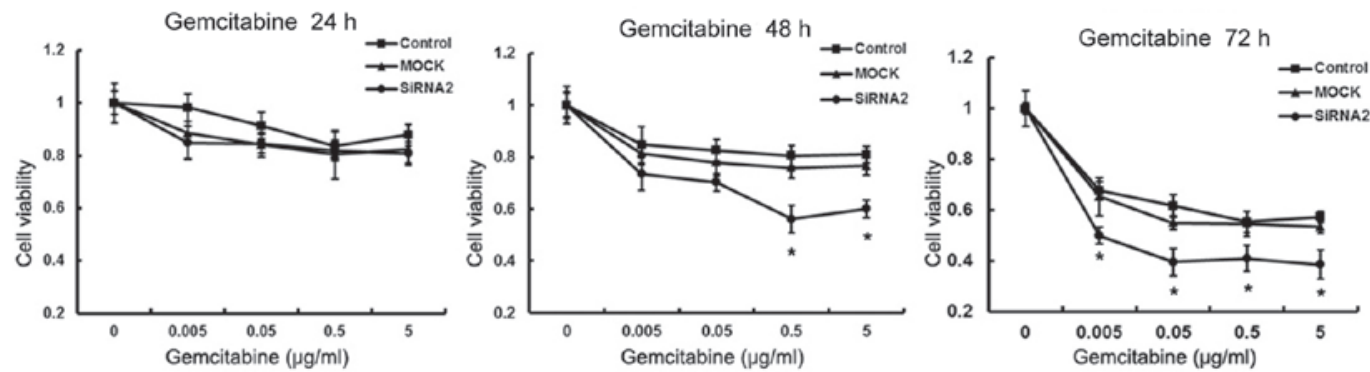

B
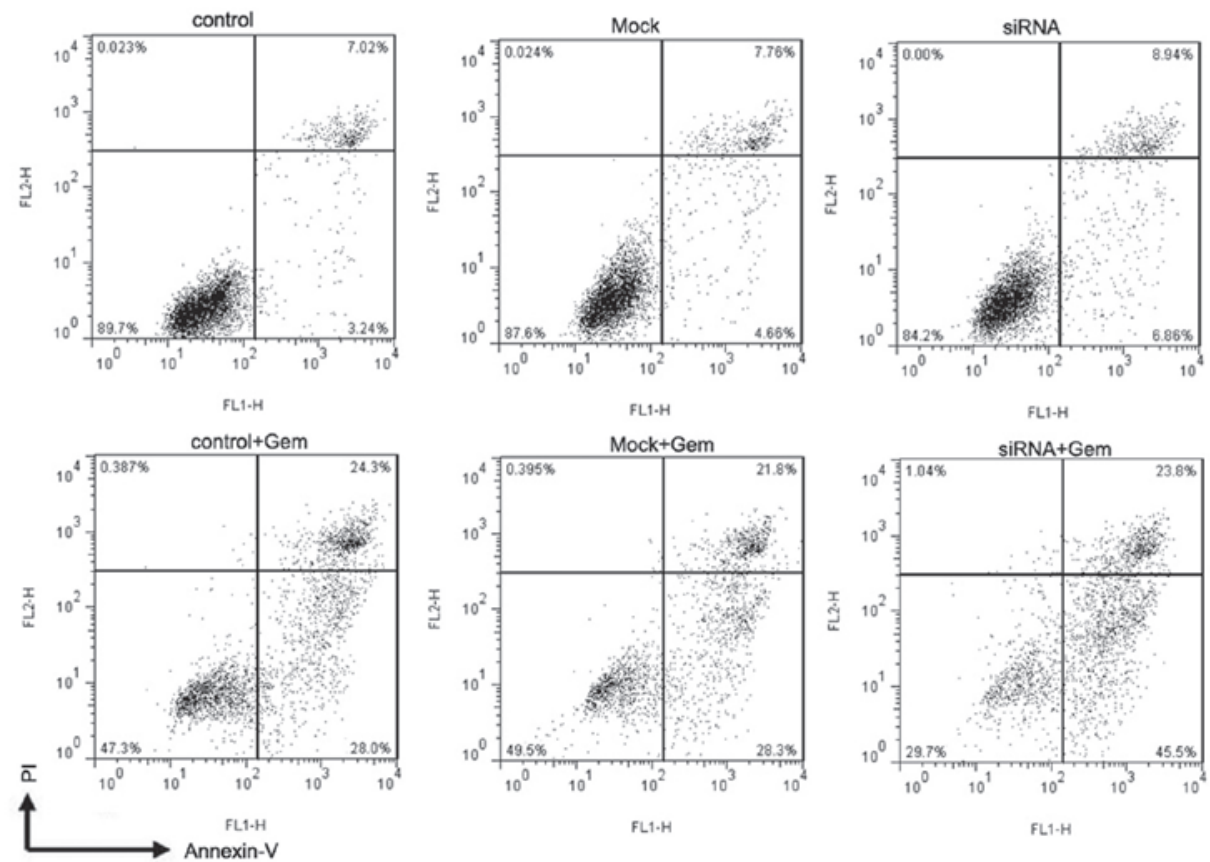

Figure 6. Effects of MACC1 suppression on the chemoresistance of pancreatic cancer cells. (A) Cells transfected with or without siRNA were treated with various concentrations $(0.005-5 \mu \mathrm{g} / \mathrm{ml})$ of gemcitabine for 24,48 and $72 \mathrm{~h}$ and then viable cells were analyzed by the MTT assay. Each data point was presented as the mean \pm SD from three independent experiments. ${ }^{*} \mathrm{P}<0.05$. (B) Flow cytometry analysis of apoptotic cells that were treated with or without gemcitabine. Gemcitabine concentration was $0.5 \mu \mathrm{g} / \mathrm{ml}$ for $48 \mathrm{~h}$. A representative result is shown. Gem, gemcitabine; MACC1, metastasis-associated in colon cancer-1; EMT, epithelial-mesenchymal transition; SD, standard deviation. 


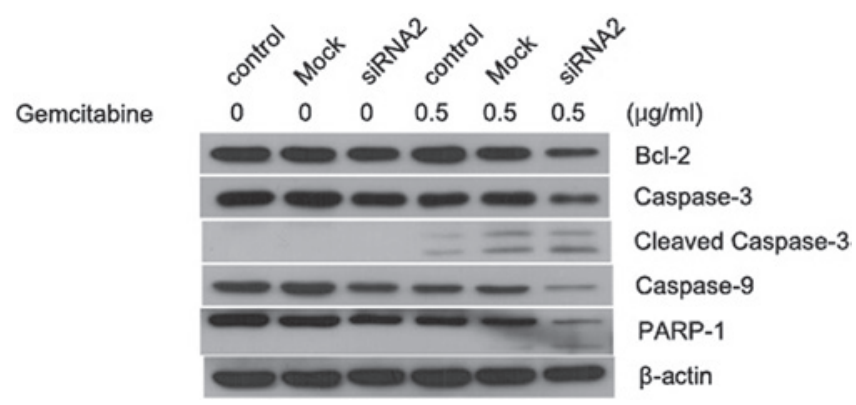

Figure 7. Effect of MACC1-siRNA on gemcitabine-induced apoptosis. CFPAC-1 cells were transfected with mock or MACC1-siRNA for $24 \mathrm{~h}$ and treated with or without gemcitabine $(0.5 \mu \mathrm{g} / \mathrm{ml})$ for $48 \mathrm{~h}$. Total protein was obtained and samples were subjected to western blot analysis for various cell apoptosis-associated proteins (Bcl-2, caspase-3, caspase-9, PARP-1). $\beta$-actin served as a loading control. MACC1, metastasis-associated in colon cancer-1.

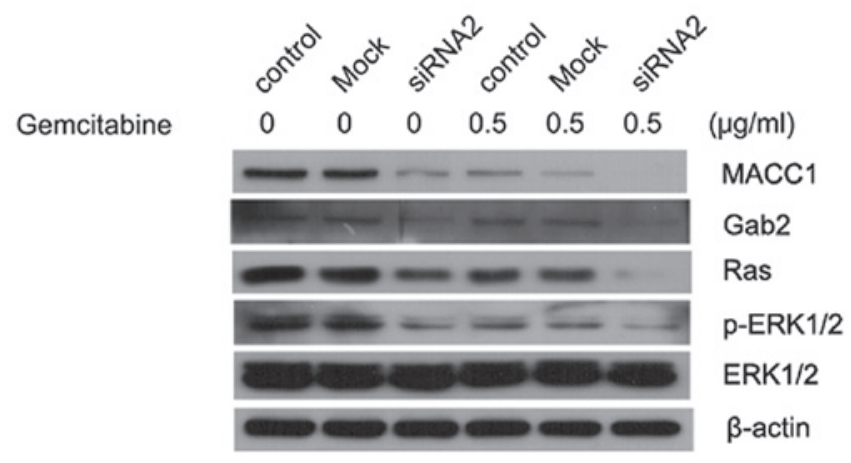

Figure 8. Effect of gemcitabine combined with MACC1-siRNA on the Ras-ERK signaling pathway. Whole lysates from CFPAC-1 cells treated as indicated in Patients and methods, were subjected to western blot analysis with antibodies against Ras, phosphor-ERK, ERK and Gab2. $\beta$-actin served as a loading control. MACC1, metastasis-associated in colon cancer-1.

respectively. The apoptotic rates of the control and mocktransfected groups at $0.5 \mu \mathrm{g} / \mathrm{ml}$ gemcitabine were 28.0 and $28.3 \%$, respectively, while the apoptotic rate in the MACC1 silenced group was $45.5 \%$ (Fig. 6B).

As revealed by immunoblotting analysis (Fig. 7), MACC1 knockdown induced significant apoptosis in CFPAC-1 cells following treatment with gemcitabine. This result was verified by a decreased expression of pro-caspase-3, pro-caspase- 9 and PRAP proteins and an increased production of cleaved caspase-3. siRNA-mediated knockdown of MACC1 also reduced the expression of $\mathrm{Bcl}-2$, an anti-apoptotic protein, following gemcitabine stimulation. Such chemosensitization effects of MACC1 knockdown were not evident without gemcitabine treatment. In normal conditions, knockdown of MACC1 alone induced slight cell apoptosis, suggesting a potential role of sensitization under stressful situations.

MACC1 regulates the Ras/ERK pathway in pancreatic cancer cells. We investigated whether MACC1 exhibited any effects on the Ras/ERK pathway. Knockdown of MACC1 decreased the expression of Ras and pERK1/2, where such effects were more evident during gemcitabine treatment (Fig. 8). Silencing MACC1 also led to the downregulation of Gab2, an adaptor protein that potentiated the activation of the Ras/ERK and $\mathrm{PI} 3 \mathrm{~K} / \mathrm{AKT}$ pathways and is associated with mammary tumorigenesis and metastasis. We found that gemcitabine treatment alone reduced the activation of Gab2, Ras and pERK, which may be attributed to the gemcitabine-mediated MACC1 downregulation. However, further studies are required to prove such correlations between MACC1 and the Ras/ERK pathway.

\section{Discussion}

MACC1 was first identified using qRT-PCR and databank analysis, as a colon cancer-associated gene, by Stein et al (6). These authors revealed that MACC1 was a prognostic marker for colon cancer metastasis and metastasis-free survival (6). Since then, the association between MACC1 levels and the various cancer risks and prognosis have been analyzed using immunohistochemistry and qRT-PCR. MACC1 has been demonstrated to be a metastatic or poor prognosis marker for colon cancer $(6,8)$, gastric cancer $(12)$, lung adenocarcinoma $(10,11)$, hepatocellular carcinoma $(9,16)$ and ovarian cancer (17).

Stein et al indicated that MACC1 was located on the human chromosome 7 (7p21.1) and its role was to markedly induce cell migration, invasion, colony formation and proliferation (6). It was observed that in metastatic lesions MACC1 was mainly expressed in the nuclei of tumor cells, while in non-metastasizing tumor cells, MACC1 was predominantly observed in the cytoplasm. Additionally, when treated with HGF, MACC1 was able to translocate to the nucleus from the cytoplasm and then bind to the Met promoter to regulate its expression. These findings demonstrated that MACC1 served as the major regulator of the $\mathrm{HGF} / \mathrm{Met}$ signaling pathway. Furthermore, Stein et al indicated that MACC1 may be involved in the MAPK signaling pathway (6).

To the best of our knowledge, this is the first study to investigate serum MACC1 expression in human pancreatic cancer patients using ELISA methods. In the present study, we found that serum MACC1 values were significantly elevated in pancreatic cancer patients, but were weakly expressed or not expressed in healthy subjects $(\mathrm{P}<0.001)$. Furthermore, we found that high levels of MACC1 often correlated with enhanced lymph node metastasis, distant metastasis and a later TNM stage. However, MACC1 serum levels had no significant associations with gender, age or depth of invasion. Therefore, serum MACC1 levels are considered to be correlated with more aggressive pancreatic cancers and may be useful for diagnosis.

The role of MACC1 in pancreatic cancer has not been reported, therefore, we examined the expression of MACC1 in two colon cancer cell lines and five pancreatic cancer cell lines. The CFPAC-1 cell line, which presented the highest level of MACC1, was selected for the subsequent experiments. In our study, we revealed that downregulation of MACC1 expression by RNA interference was able to suppress the proliferation and migration of pancreatic cancer cells, as well as enhance gemcitabine-induced cytotoxicity.

EMT was first identified as a culmination of transcriptional and protein modification events in early embryonic morphogenesis (18). Emerging evidences indicated that the EMT was a developmental process that involved the reduction 
of intercellular adhesions and the acquisition of mesenchymal cell characteristics. EMT plays a significant role in tumor metastasis, invasion and carcinogenesis (19). Previous studies have demonstrated that gemcitabine-resistant pancreatic cancer cells acquired the EMT phenotype, and that EMT may maintain drug resistance in human pancreatic cancer cells (20-22). In the present study, we found that the suppression of MACC1 not only inhibits the migration of CFPAC-1 cells, but also causes the upregulation of E-cadherin, a marker for epithelial cell phenotype. We revealed for the first time that inhibiting MACC1 reversed the EMT phenotype and may increase drug sensitivity. However, other EMT markers should be investigated to confirm the results.

The Ras/ERK pathway is involved in the regulation of a variety of biological functions, including cell proliferation, survival and apoptosis. In addition to this, the Ras/ERK pathway has been identified to be mutated in approximately $30 \%$ of all types of cancer, with the highest incidences found in pancreatic cancer (90\%) (23). Ras proteins are distributed in a variety of plasma membrane microdomains and translocated between cell compartments (24). Activated K-ras mutations are considered to be the initial step of pancreatic ductal carcinogenesis (25), while the knockdown of K-ras by RNA interference enhances gemcitabine-induced apoptosis in pancreatic cancer (26). In the present study, suppression of MACC1 was able to inhibit the growth of CFPAC-1 cells, and induce enhanced apoptosis when combined with gemcitabine treatment. We demonstrated the induction of apoptotic cell death using multiple assays, including MTT, flow cytometry and western blot analysis. We also found that the suppression of MACC1 combined with gemcitabine significantly inhibited the activity of the Ras/ERK signaling pathway. This was identified by investigating several downstream targets of the Ras/ERK pathway, including Bcl-2, caspase-3 and caspase-9. It was also found that treatment with MACC1-siRNA significantly decreased the expression of Gab2, a member of the Gab family of scaffolding adaptors that also contains Gab1 and Gab3 (27). The majority of Gab protein-receptor interactions are mediated indirectly via Grb2, and the direct recruitment has been demonstrated only between Gab1 and c-Met (28). Previous studies have demonstrated that Gab-Shp2 complexes act as 'amplifiers' of the Ras/ERK signaling pathway activation (29-30). Therefore, we considered that the Gab family may be involved in the process of the MACC1-mediated activation of the Ras/ERK signaling pathway, but the clear mechanisms require further investigation.

In this study, we present evidence that MACC1 overexpression is detectable in the serum of pancreatic cancer patients and correlated with tumor metastasis. Therefore, serum MACC1 expression has value in the diagnosis of pancreatic cancer. However, the sample size used in our study is small and investigations should be conducted to confirm our findings in a larger cohort of patients. MACC1 may also play a significant role in chemoresistance through regulating the Ras/ERK signaling pathway. In conclusion, MACC1 may prove to be a novel therapeutic target for gemcitabine treatment; however, ongoing studies are required to fully explore the molecular mechanisms involved in tumor metastasis and chemoresistance.

\section{Acknowledgements}

This study was supported by grants from the National Natural Science Foundation of China (nos. 81172158, 81001094, 81071960, 30901445 and 81100549).

\section{References}

1. Jemal A, Siegel R, Xu J and Ward E: Cancer statistics. CA Cancer J Clin 60: 277-300, 2010.

2. Jemal A, Bray F, Center MM, Ferlay J, Ward E and Forman D: Global cancer statistics. CA Cancer J Clin 61: 69-90, 2011.

3. Pliarchopoulou K and Pectasides D: Pancreatic cancer: Current and future treatment strategies. Cancer Treat Rev 35: 431-436, 2009.

4. Maitra A and Hruban RH: Pancreatic cancer. Annu Rev Pathol 3: 157-188, 2008

5. Shen J, Person MD, Zhu J, Abbruzzese JL and Li D: Protein expression profiles in pancreatic adenocarcinoma compared with normal pancreatic tissue and tissue affected by pancreatitis as detected by two-dimensional gel electrophoresis and mass spectrometry. Cancer Res 64: 9018-9026, 2004.

6. Stein U, Walther W, Arlt F, et al: MACC1, a newly identified key regulator of HGF-MET signaling, predicts colon cancer metastasis. Nat Med 15: 59-67, 2009.

7. Arlt F and Stein U: Colon cancer metastasis: MACC1 and Met as metastatic pacemakers. Int J Biochem Cell Biol 41: 2356-2359, 2009.

8. Shirahata A, Shinmura K, Kitamura Y, et al: MACC1 as a marker for advanced colorectal carcinoma. Anticancer Res 30: 2689-2692, 2010.

9. Shirahata A, Fan W, Sakuraba K, et al: MACC 1 as a marker for vascular invasive hepatocellular carcinoma. Anticancer Res 31: 777-780, 2011.

10. Chundong G, Uramoto H, Onitsuka T, et al: Molecular diagnosis of MACC1 status in lung adenocarcinoma by immunohistochemical analysis. Anticancer Res 31: 1141-1145, 2011.

11. Shimokawa H, Uramoto H, Onitsuka T, et al: Overexpression of MACC1 mRNA in lung adenocarcinoma is associated with postoperative recurrence. J Thorac Cardiovasc Surg 141: 895-898, 2011.

12. Shirahata A, Sakata M, Kitamura Y, et al: MACC 1 as a marker for peritoneal-disseminated gastric carcinoma. Anticancer Res 30: 3441-3444, 2010.

13. Sobin LH and Wittekind WC (eds): TNM Classification of Malignant Tumours (UICC). 6th edition. Wiley-Liss, New York, pp93-96, 2002.

14. Altman DG, Lausen B, Sauerbrei W and Schumacher M: Dangers of using 'optimal' cutpoints in the evaluation of prognostic factors. J Natl Cancer Inst 86: 829-835, 1994.

15. Arlt A, Gehrz A, Muerkoster S, et al: Role of NF-kappaB and $\mathrm{Akt} / \mathrm{PI} 3 \mathrm{~K}$ in the resistance of pancreatic carcinoma cell lines against gemcitabine-induced cell death. Oncogene 22: 3243-3251, 2003.

16. Qiu J, Huang P, Liu Q, et al: Identification of MACC1 as a novel prognostic marker in hepatocellular carcinoma. J Transl Med 9: $166,2011$.

17. Zhang R, Shi H, Chen Z, Wu Q, Ren F and Huang H: Effects of metastasis-associated in colon cancer 1 inhibition by small hairpin RNA on ovarian carcinoma OVCAR-3 cells. J Exp Clin Cancer Res 30: 83, 2011.

18. Shook D and Keller R: Mechanisms, mechanics and function of epithelial-mesenchymal transitions in early development. Mech Dev 120: 1351-1383, 2003.

19. Yang J and Weinberg RA: Epithelial-mesenchymal transition: at the crossroads of development and tumor metastasis. Dev Cell 14: 818-829, 2008.

20. ShahAN, Summy JM,Zhang J, Park SI, Parikh NU and Gallick GE: Development and characterization of gemcitabine-resistant pancreatic tumor cells. Ann Surg Oncol 14: 3629-3637, 2007.

21. Wang Z, Li Y, Kong D, et al: Acquisition of epithelial-mesenchymal transition phenotype of gemcitabine-resistant pancreatic cancer cells is linked with activation of the notch signaling pathway. Cancer Res 69: 2400-2407, 2009.

22. Arumugam T, Ramachandran V, Fournier KF, et al: Epithelial to mesenchymal transition contributes to drug resistance in pancreatic cancer. Cancer Res 69: 5820-5828, 2009. 
23. Bos JL: Ras oncogenes in human cancer: a review. Cancer Res 49: 4682-4689, 1989.

24. Calvo F, Agudo-Ibanez L and Crespo P: The Ras-ERK pathway: understanding site-specific signaling provides hope of new antitumor therapies. Bioessays 32: 412-421, 2010.

25. Deramaudt T and Rustgi AK: Mutant KRAS in the initiation of pancreatic cancer. Biochim Biophys Acta 1756: 97-101, 2005.

26. Rejiba S, Wack S, Aprahamian M and Hajri A: K-ras oncogene silencing strategy reduces tumor growth and enhances gemcitabine chemotherapy efficacy for pancreatic cancer treatment. Cancer Sci 98: 1128-1136, 2007.

27. Nishida K and Hirano T: The role of Gab family scaffolding adapter proteins in the signal transduction of cytokine and growth factor receptors. Cancer Sci 94: 1029-1033, 2003.
28. Gu H and Neel BG: The 'Gab' in signal transduction. Trends Cell Biol 13: 122-130, 2003.

29. Yart A, Laffargue M, Mayeux P, et al: A critical role for phosphoinositide 3-kinase upstream of Gab1 and SHP2 in the activation of ras and mitogen-activated protein kinases by epidermal growth factor. J Biol Chem 276: 8856-8864, 2001.

30. Cunnick JM, Meng S, Ren Y, et al: Regulation of the mitogen-activated protein kinase signaling pathway by SHP2. J Biol Chem 277: 9498-9504, 2002. 